\title{
Integrated Attitude Control Strategy for the Asteroid Redirect Mission
}

\author{
Pedro Lopez Jr. ${ }^{1}$ \\ NASA Johnson Space Center (EA3), Houston, TX 77058 \\ Hoppy Price ${ }^{2}$ and Miguel San Martin ${ }^{3}$ \\ NASA Jet Propulsion Laboratory (312E), Pasadena, CA 91109
}

\begin{abstract}
A deep-space mission has been proposed to redirect an asteroid to a distant retrograde orbit around the moon using a robotic vehicle, the Asteroid Redirect Vehicle (ARV). In this orbit, astronauts will rendezvous with the ARV using the Orion spacecraft. The integrated attitude control concept that Orion will use for approach and docking and for mated operations will be described. Details of the ARV's attitude control system and its associated constraints for redirecting the asteroid to the distant retrograde orbit around the moon will be provided. Once Orion is docked to the ARV, an overall description of the mated stack attitude during all phases of the mission will be presented using a coordinate system that was developed for this mission. Next, the thermal and power constraints of both the ARV and Orion will be discussed as well as how they are used to define the optimal integrated stack attitude. Lastly, the lighting and communications constraints necessary for the crew's extravehicular activity planned to retrieve samples from the asteroid will be examined. Similarly, the joint attitude control strategy that employs both the Orion and the ARV attitude control assets prior, during, and after each extravehicular activity will also be thoroughly discussed.
\end{abstract}

\section{Nomenclature}

$\begin{array}{ll}A C S & =\text { attitude control subsystem } \\ A R C M & =\text { asteroid redirect crewed mission } \\ A R V & =\text { asteroid redirect vehicle } \\ B E O & =\text { beyond Earth orbit } \\ C M & =\text { center of mass, Crew Module (Orion) } \\ C P & =\text { center of pressure } \\ D C & =\text { docking camera } \\ D R M & =\text { design reference mission } \\ D R O & =\text { distant retrograde orbit } \\ D S N & =\text { deep-space network } \\ E V A & =\text { extra-vehicular activity } \\ I D D & =\text { interface definition document } \\ I D S S & =\text { international docking system standard } \\ I M U & =\text { inertial measurement unit } \\ L O C I & =\text { laser optical camera instrument } \\ M E C O & =\text { main engine cut-off } \\ N D S & =\text { NASA docking system } \\ P P U & =\text { power processing unit } \\ R C S & =\text { reaction control system } \\ R W A & =\text { reaction wheel assembly } \\ S E P & =\text { solar electric propulsion }\end{array}$

${ }^{1}$ Engineer, Systems Engineering Management Branch, Engineering Directorate Office.

${ }^{2}$ Supervisor, Pre-Projects Systems Engineering Group.

${ }^{3}$ Chief Engineer, Guidance and Control Section. 


$\begin{array}{ll}\text { SLS } & =\text { Space Launch System } \\ \text { SM } & =\text { Service Module }(\text { Orion) } \\ \text { SMAP } & =\text { Soil Moisture Active/Passive } \\ V N S & =\text { vision navigation sensor }\end{array}$

\section{Introduction}

$\mathrm{T}$ IIS paper provides detailed information on the assets and strategies assumed in the Asteroid Redirect Crewed Mission (ARCM) to perform attitude control of the various vehicles, with an emphasis on those used for the integrated Asteroid Redirect Vehicle/Orion stack. This mission assumes that an asteroid, previously identified and thoroughly studied via ground observations and/or previous robotic missions, has been successfully redirected to a given orbit in the vicinity of the moon. First, a short description of the ARCM scenario as well as the assets required on the ARV and Orion to perform docked operations is provided. A review of the ARV attitude control system is also reviewed. This is followed by a discussion on the integrated attitude control strategy, including the attitudes selected for docking and undocking, as well as for EVA operations based on spacecraft limitations due to thermal and power constraints and mission requirements such as worksite lighting conditions and vehicle-to-ground communication.

\section{A. ARCM Design Reference Mission}

The ARCM Design Reference Mission (DRM) centers on the translunar transit, rendezvous, docking, and earth return of an SLS-launched Orion spacecraft carrying two crew members to an asteroid previously captured and redirected to a Distant Retrograde Orbit (DRO) by the ARV. A DRO is a highly stable orbit that was selected for long-term stability, Orion-SLS access and launch availability. DROs are stable orbits where an object can be "parked" and remain in orbit for over one hundred years with minimal orbit maintenance maneuvers required. This stability is attractive to ensure the asteroid remains in place while minimizing the orbit maintenance propulsion required for the ARV.

Several factors limit opportunities to launch to the DRO. Launch opportunities for the Orion-SLS are subject to several constraints including, but not limited to: launch epoch, trajectory state at Main Engine Cut Off (MECO state), upper stage performance capability, Orion performance capability and the ARV phase angle in the DRO at the time of Orion Earth orbit departure. The mission timelines for some launch opportunities will have multiple days of communication dropouts caused by the combination of mission attitude requirements and blockage caused by the ARV solar arrays and the asteroid. Finally, power generation requirements dictate that the docked vehicles avoid timeframes when the Earth and/or Moon eclipses the sun. Despite these constraints, initial analysis of a representative epoch showed approximately two launch opportunities in any given month with acceptable constraints on trajectory, communications coverage, and eclipse periods.

\section{B. Baseline Orion Configuration}

The Orion is a pressurized, crewed element that transports up to four crew members from the Earth's surface to Beyond Earth Orbit (BEO) destinations or staging points and returns the crew members safely back to the Earth's surface at the end of a mission. The Orion provides all services necessary to support the crew members for short duration missions, or until Orion docks with another vehicle to augment crew consumables and enable longer duration missions.

The reference Orion configuration used for this assessment is based on the BEO Orion for the EM-2 Lunar Orbit mission, as defined in MPCV-72093, MPCV Program ConOps. The EM-2 Orion includes full crew life support systems. The baseline EM-2 mission configuration supports a crew of four for a 21 day mission duration. The crew size and mission duration can be adjusted by changing the vehicle configuration.

The EM-2 Orion does not support EVA. Reducing the crew complement from four to two provides additional internal stowage and mass capability. This recovered volume allows for the addition of ARCM "mission kits" which will extend the capability of the Orion to support the ARCM flight. The fundamental design guideline for the kits is to provide the necessary functions self-contained within the kits with minimal changes to the baseline Orion EM-2 configuration and ground support equipment. The two kits that are directly related to the integrated attitude control function are the relative navigation kit and the docking kit. 
The relative navigation kit will add a Laser Optical Camera Instrument (LOCI) to supply the measurements for relative navigation. LOCI is comprised of two Vision Navigation Sensors (VNS) and two Docking Cameras (DC). The VNS component provides range and bearing from six $\mathrm{km}$ to dock, and then provides relative position and relative attitude from 15 meters to dock. The DCs provide relative bearing from 15 $\mathrm{km}$ to dock, and situational awareness to the crew performing docking operations. A notional concept of LOCI mounted to the Orion's docking hatch is shown in Fig. 1. The Orion will have docking lights on the exterior side of the docking hatch to provide target illumination.

The NASA Docking System (NDS) is assumed to represent the docking interface for mating the Orion to the ARV. The NDS is comprised of an active mechanism on the Orion and a passive mechanism on the ARV. The NDS

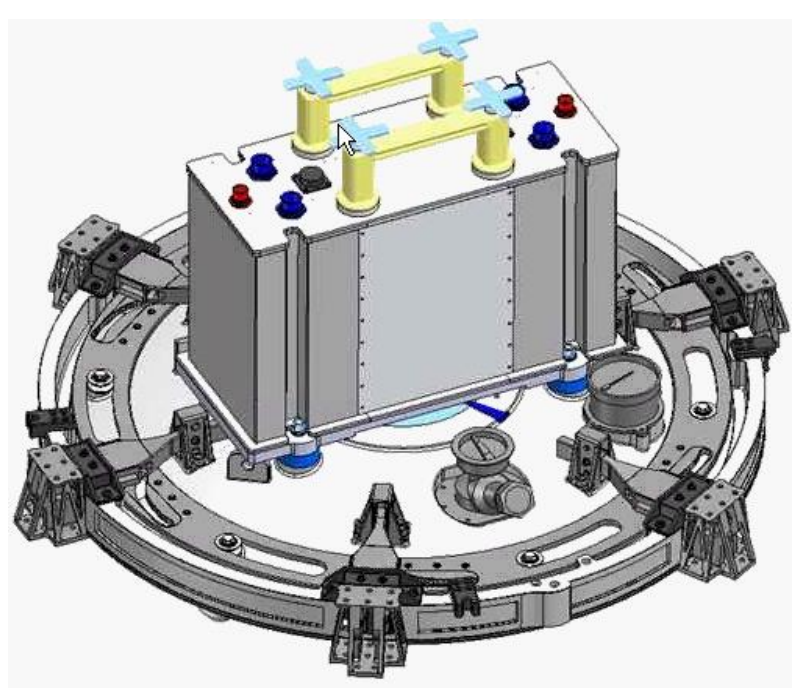

Figure 1. LOCI mounted to Orion docking hatch (interior view) active mechanism will be attached to the Orion structural ring that will be used as the docking mechanism interface. The NDS will comply with the International Docking System Standard Interface Definition Document (IDSS IDD). Figure 2 shows details of the IDSScompliant docking interface. Orion will supply power to the NDS and receive data on NDS performance. The NDS specifications include connectors for power and data transfer between the Orion and the ARV. The ARCM

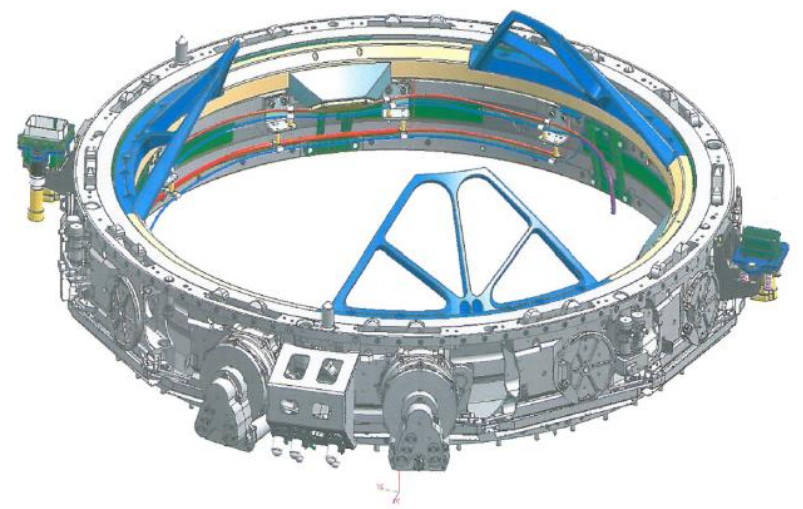
flight does not require the power or data transfer capability through the docking kit at this time, though that capability will be highly desirable in subsequent missions to the ARV. Since the ARV does not contain a pressurized segment, the hatchway in the NDS specification is not required for the ARCM. Similarly, there is no requirement for the utility connections in the NDS specification. The interface between the Docking Kit and the Orion will allow for jettison of the docking mechanism prior to re-entry.

Figure 2. IDSS-Compliant Docking Interface

Table 1 lists some of the functions provided by Orion in support of ARCM, including those available via the mission kits. 
Table 1. Orion Functions and Capabilities

\begin{tabular}{|c|c|}
\hline Functional Requirement & Spacecraft Capabilities \\
\hline Provide Crew Habitation & $\begin{array}{l}4 \text { crew for } 21 \text { days (EM-2 specification limit) } \\
2 \text { crew for } 28 \text { days (ARCM design) }\end{array}$ \\
\hline Provide EVA Capability & $\begin{array}{l}\text { Orion provides one cabin air repressurization to } \\
\text { support a contingency EVA in suits constrained to } \\
\text { umbilicals. } \\
\text { ARCM adds two additional represses to support two } \\
\text { planned EVAs off umbilicals using an Exploration } \\
\text { Portable Life Support System (Exploration PLSS) } \\
\text { service equipment and tools }\end{array}$ \\
\hline Provide Sample Return Capability & ARCM adds Sample Return Kit \\
\hline $\begin{array}{l}\text { Accomplish Rendezvous, Docking, and } \\
\text { Undocking }\end{array}$ & $\begin{array}{l}\text { Orion provides crew windows, crew-in-the-loop flight } \\
\text { control and star trackers. } \\
\text { ARCM adds docking mechanism and } \\
\text { rendezvous/prox-ops sensors. }\end{array}$ \\
\hline Provide Electrical Power & 4 Solar arrays, batteries \\
\hline Provide Communication to Ground & 8 S-band antennas on Orion CM and SM \\
\hline Provide Thermal Control & Radiator side panels on SM \\
\hline Provide Propulsion & $\begin{array}{l}1 \text { biprop main engine } \\
8 \text { auxiliary engines } \\
24 \text { attitude thrusters }\end{array}$ \\
\hline
\end{tabular}

\section{ARV Configuration}

Details of the overall Asteroid Redirect Vehicle (ARV) configuration can be found in various JPL publications related to the asteroid redirect mission. This section describes the aspects of the ARV configuration that support the ARCM, and more specifically, prox ops, docking, and integrated attitude control. Figure 3 shows the ARV configuration being considered in this feasibility study.

The ARV will require the addition of up to three small retro-reflector assemblies and a S-Band system to support proximity operations with the Orion vehicle. A passive docking mechanism will be added to the aft end of the ARV, opposite the asteroid capture mechanism, to facilitate docking with Orion. The docking mechanism will contain a docking target for Orion relative navigation sensors and crew situational awareness. This study assumes the docking mechanism is derived from an IDSScompliant, passive NDS. As currently defined, the NDS contains connectors for transmission of power and data through the mechanism. Neither the hardline power nor data function is required for the ARCM, but will provide a significant resource for

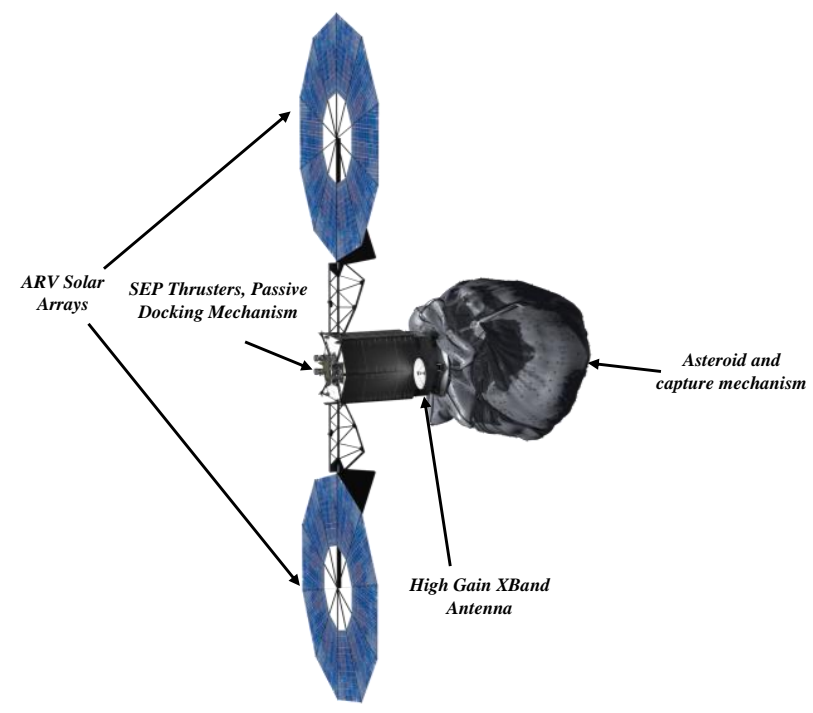

Figure 3. ARV Concept showing passive NDS and high-gain antenna extensibility in the future.

\section{Vehicle-to-Vehicle Communications}

Orion will use the same Vehicle to Ground Communication Path established in support of EM-1 and EM-2. Currently, that capability is an S-band system direct from the Orion to Earth via the Deep Space Network (DSN) and supports real-time mission command and telemetry, ascent/entry command and telemetry to support launch, aborts, downlink of real time data/video to support operations, catastrophic failure analysis, recovery needs, education and public outreach objectives, and flight certification elements. On the other hand, the ARV will use an X-Band system 
for vehicle to ground communications, also utilizing the DSN. An S-band system will be added to the ARV design to facilitate vehicle-to-vehicle communication during rendezvous operations. Figure 4 shows an overview of the communication systems for the two vehicles.

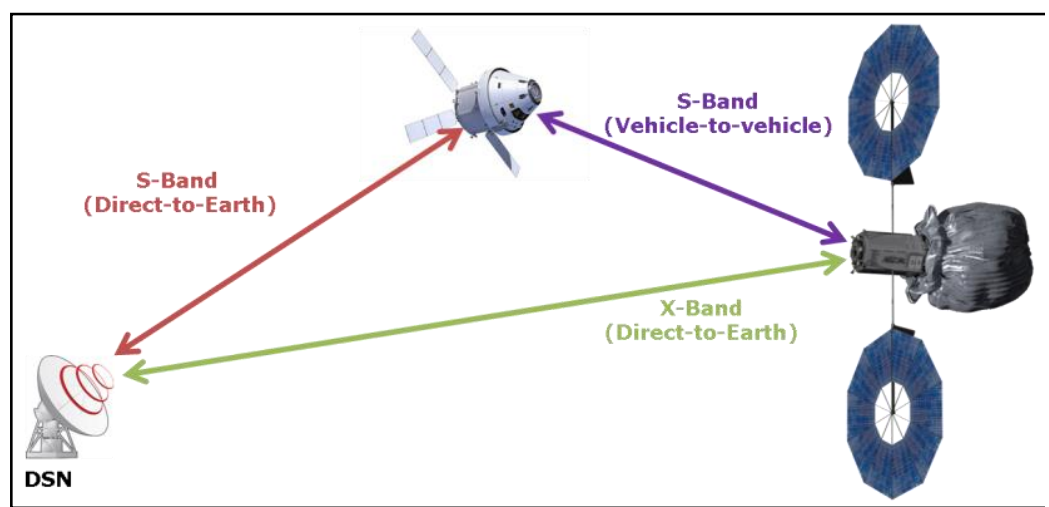

Figure 4. ARCM Communication Overview

\section{ARV Attitude Control Subsystem}

The ARV is 3-axis stabilized. While in powered Solar Electric Propulsion (SEP) flight, the four operating gimbaled Hall thrusters provide pitch, yaw, and roll control of the ARV. While not in powered flight, turning maneuvers are provided by the hydrazine Reaction Control System (RCS) thrusters. Figure 5 provides a close-up view of the aft side of the ARV, showing the SEP thrusters as well as the RCS thrusters. When not maneuvering, the four Soil Moisture Active/Passive (SMAP) Program heritage Reaction Wheel Assemblies (RWAs) are used to hold the ARV in a highly stabilized position. The RWAs can also be used for slow maneuvers prior to asteroid capture. The RWAs, star trackers, Inertial Measurement Units (IMUs), and sun sensors are all SMAP heritage components. The IMUs also contain accelerometers that will be utilized for maneuvering around the asteroid and during the capture phase. The RWAs are mounted in a pyramid configuration as they are on SMAP.

While thrusting using the $40 \mathrm{kWe}$ SEP system, the sun is generally restricted to the plane normal to the solar array articulation axis (normal to the boom axis) for thermal control reasons, to provide a clear view to cold space for the SEP Module PPU radiators. When not thrusting with the SEP system, for example during Orion crewed visits in DRO, there are no restrictions on sun orientation for the ARV.

After the asteroid is delivered to the final

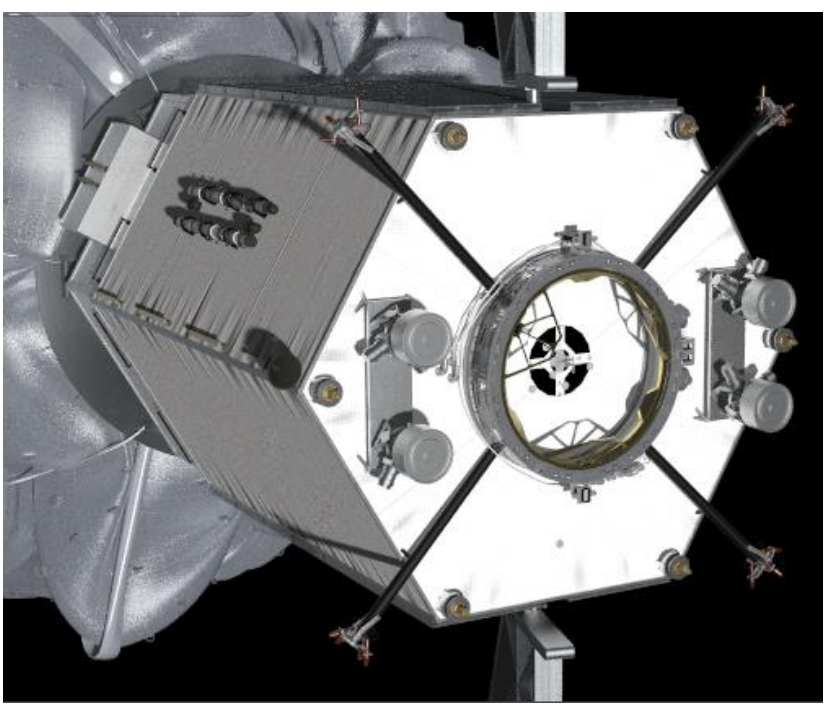

Figure 5. Close-Up View of Aft side of ARV DRO, a propellantless attitude control strategy will be utilized for stabilization of the ARV and the asteroid. The ARV will be oriented so that the Hall thrusters and the Orion docking ring are facing the sun, and asteroid is on the anti-sun side of the spacecraft. The orientation will be adjusted through a series of calibration exercises so that the center of pressure (CP) of the ARV/asteroid system is lined up with the center of mass (CM) of the ARV/asteroid system. Since the CP will be forward of the CM, this is only a quasi-stable orientation and is not a self-restoring position. This position will be held with the RWAs without the use of the hydrazine RCS thrusters.

The ARV/asteroid system will be slightly reoriented using the RWAs such that the CP is offset from the CM a small amount in the preferred direction to apply an unloading torque to the system to unload the RWAs before they 
become saturated. The solar arrays will be tilted in opposite directions to act as a solar pressure windmill and apply an unloading torque about the axis along the sunline to unload the RWAs about the axis along the sunline. Autonomous ACS software algorithms will be used to manage the wheel torques without using propellant.

For crewed operations with the Orion docked, the propellantless ACS strategy described above can also be utilized for the combined Orion/ARV/asteroid for a steady state orientation with the Orion Service Module pointed at the sun (for attitude holding). For EVA operations, the solar inertial attitude would not provide the lighting and thermal conditions required at the EVA worksite, and a propulsive maneuver would be required to adjust the attitude of the integrated stack. However, the ARV propulsion assets cannot be utilized since they could damage the Orion thermal protection system. In addition, the propellantless ACS approach cannot be utilized so the stack orientation will be controlled by the Orion attitude control assets. The strategy assessed to perform the integrated attitude control prior, during, and after EVA operations is described in the next section.

\section{Integrated Attitude Control Strategy}

\section{A. Docking and Mating Attitude}

For the purposes of the ARCM feasibility study, a coordinate system has been developed for the Orion ARV mated stack based on the Orion GN\&C coordinate frame outlined in CxP 70138 as shown in Figure 6. The X-axis extends along the length of the mated stack with positive $\mathrm{X}$ pointing from the Orion Service Module (SM) through the apex of the Orion Crew Module (CM) to the ARV. The positive $\mathrm{Y}$-axis extends through the Orion EVA hatch and the Z-axis is defined via the right hand rule, pointing opposite the crew windows of the Orion. Thus, the $\mathrm{Z}$-axis aligns with the ARV solar array rotational axis, the $\mathrm{X}$-axis extends on the ARV centerline from the Solar Electrical Propulsion (SEP) thrusters towards the asteroid and the Y-axis completes the right hand rule, extending opposite the ARV high gain antenna. The nominal mated attitude for the combined Orion ARV stack orients the X-Y plane of the mated stack configuration in the ecliptic to allow for

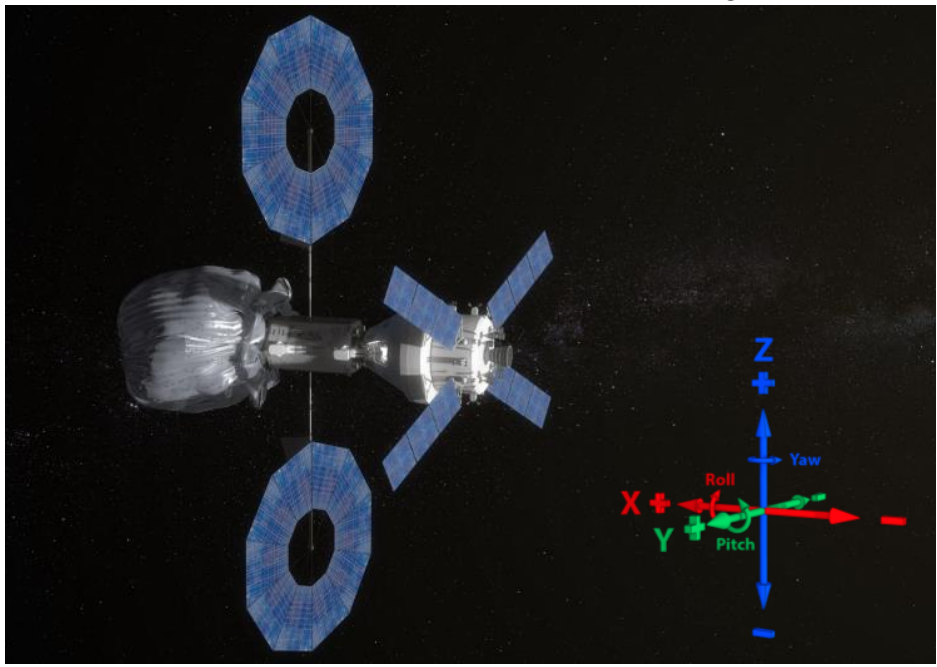

Figure 6. ARCM Coordinate Reference Frame optimal power generation while remaining within the design limits of the Orion, which require the negative $\mathrm{X}$-axis of the stack be oriented toward the sun.

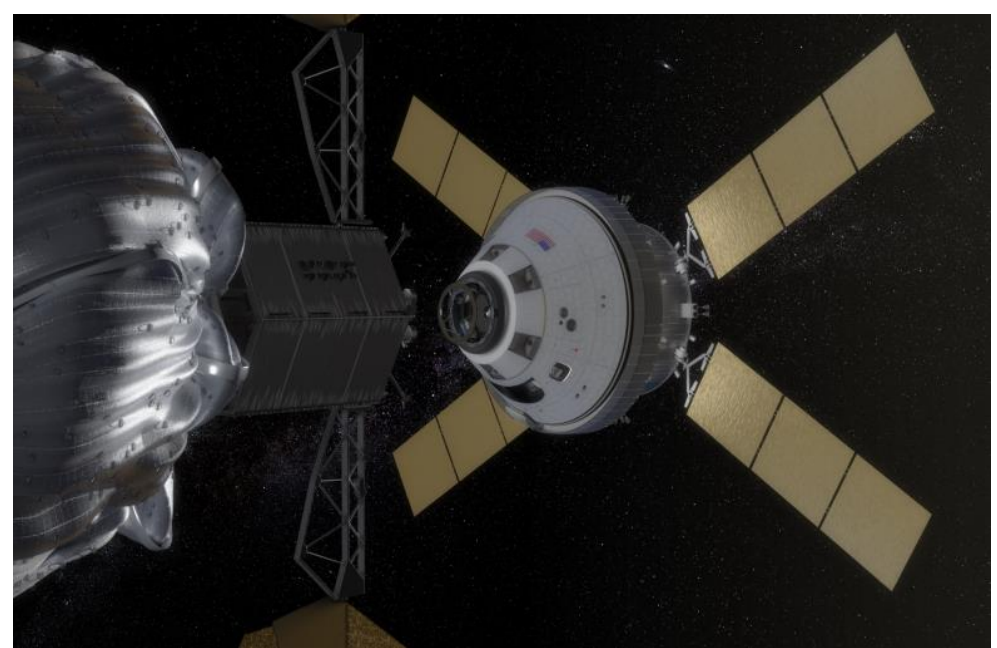

Figure 7. Orion approaching ARV prior to docking

Analysis is in work to determine the limits on departing from this default attitude. Initial results indicate Orion thermal capability will likely provide the largest constraint on a bias from the pure solar inertial attitude described above, but a bias of $0-20^{\circ}\left( \pm 20^{\circ}\right)$ should be allowable for Orion for the six-day period of mated operations.

Prior to Orion arrival at the DRO, the ARV is assumed to be at a solar inertial attitude, with either the asteroid or the ARV bus pointing at the sun depending on which attitude provides better communication coverage. If the pre-dock attitude is different from the ARCM mated attitude, the ARV will maneuver to the planned mated attitude and perform reaction wheel desaturation prior to rendezvous. Figure 7

6

American Institute of Aeronautics and Astronautics 
shows Orion approaching the ARV prior to docking, with the ARV in the solar inertial attitude.

\section{B. EVA Operations Attitude}

In the mated attitude, the Orion will cast a shadow on much of the EVA translation path along the ARV bus (see Fig. 8). The EVA suits cannot sustain lengthy dwell times in shade, so shadowing along the primary translation path is undesirable. Within the bias range allowed by preliminary Orion analysis, a bias of approximately $15^{\circ}$ yaw provides sufficient sun on the EVA worksite, specifically the ARV EVA toolbox, which will allow for longer in-suit dwell times in that location. Therefore, the initial attitude assumptions for the stack include a $15^{\circ}$ yaw during EVA operations. Figure 9 shows a representation of the light and shadow in the selected EVA attitude.

The ARV SEP and RCS thrusters cannot be used to modify the integrated attitude since Orion is docked directly behind them. In addition, the RWAs can only be used for holding a given attitude, and not for attitude maneuvers. The plan developed for analysis calls for the Orion to maneuver the mated stack from the nominal attitude to the biased solar inertial $\left(+15^{\circ}\right.$ yaw $)$ attitude to support EVA operations and back to the nominal attitude after each EVA.

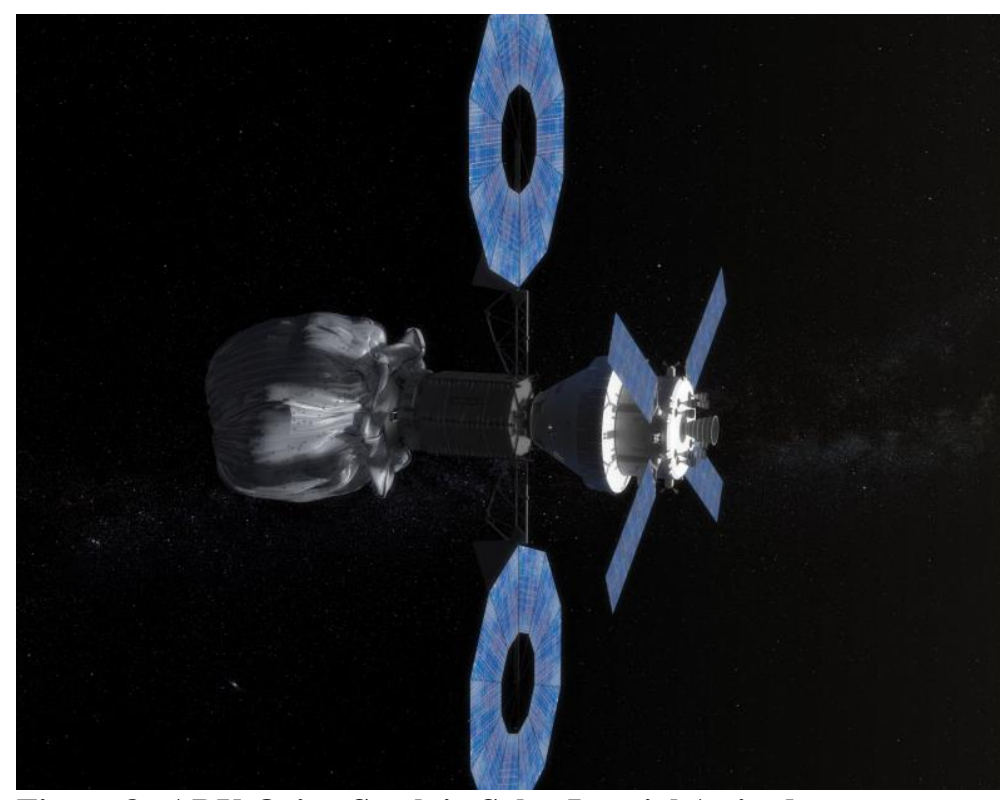

Figure 8. ARV-Orion Stack in Solar Inertial Attitude

Prior to each EVA, the Orion RCS thrusters will be used to slew the stack to $a+15^{\circ}$ yaw. This attitude change will provide improved illumination of the EVA worksite as well as the thermal conditions required for EVA operations. The Orion thrusters will then arrest

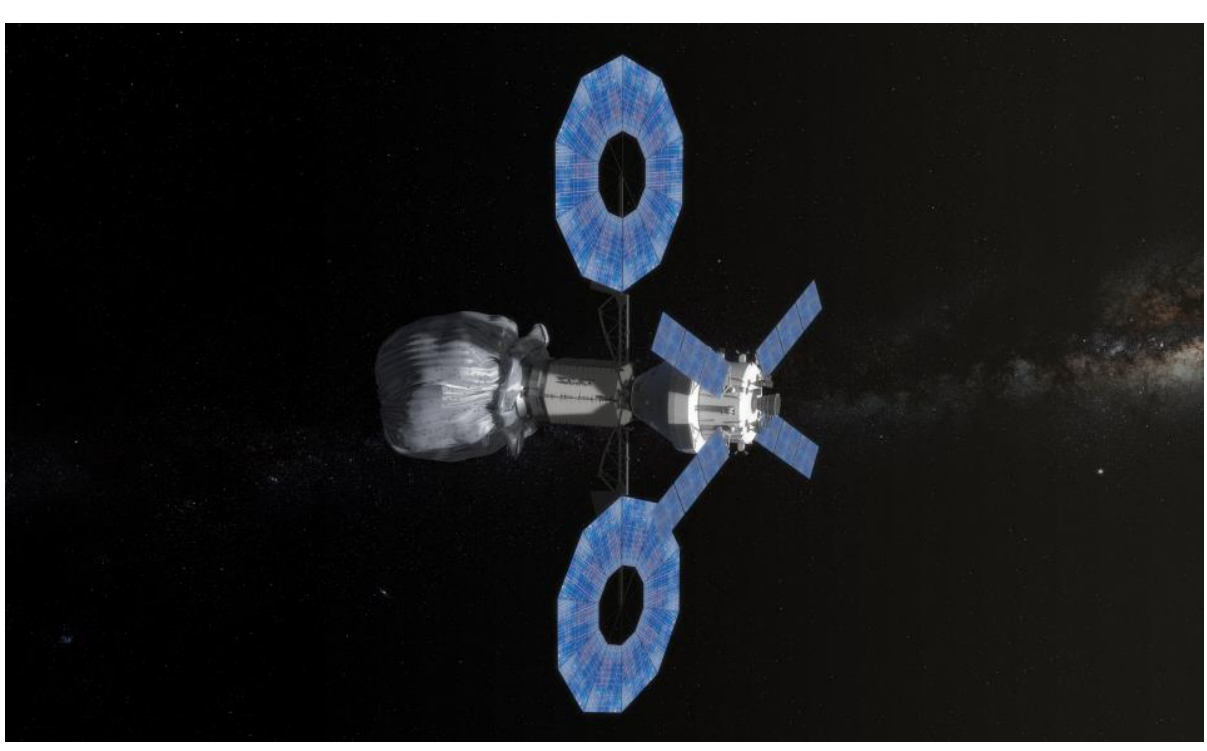

Figure 9. ARV-Orion Stack in EVA attitude any rates before the vehicle is moded to free drift for the duration of the EVA operations.

Analysis is in work to determine the ability of the ARV reaction wheels to maintain the biased stack attitude for the duration of the EVA. Ideally, the ARV reaction wheels will maintain each of those attitudes in order to meet EVA constraints and conserve propellant.

Once all EVA operations have been completed, and the crew is safely back in Orion (repressed) with suits doffed, it is assumed that the Orion RCS thrusters are used to return the stack to its nominal solar inertial attitude as well as arrest any residual rates. Once the nominal attitude is achieved, the ARV reaction wheels will resume stack attitude maintenance. This process will be repeated for every EVA operation to maximize power generation and reduce propellant usage. 
On the day prior to undocking from the ARV, the docking mechanism will be exercised by issuing commands through each redundant string to perform checkout of the various system components. The ARV will be moded to free drift prior to undocking and will remain in free drift until the Orion is a sufficient distance away. Once Orion has reached a predetermined distance from the ARV, the RWAs will resume control of the ARV and bring it back to its nominal solar inertial attitude.

\section{Conclusion}

A strategy to address integrated attitude control of the ARV/Orion/asteroid stack in support of the ARCM has been assessed. Docking of Orion to the aft of the ARV precludes the use of the SEP and RCS thrusters for integrated attitude control to avoid damaging the Orion thermal protection system. The strategy calls for use of the Orion RCS thrusters to maneuver the stack to the solar inertial and $+15^{\circ}$ yaw attitudes for nominal and EVA operations, respectively, as well as to arrest any residual rates. These two attitudes are within the thermal and power constraints of the ARV as well as Orion. A propellantless attitude control strategy will be utilized to hold the ARV/Orion/asteroid stack at each attitude using the ARV reaction wheel assembly. Analyses are still being

performed, however initial review shows that the current attitude control strategy can be used to successfully complete all ARCM objectives.

\section{Acknowledgments}

The authors would like to acknowledge the support of the Asteroid Redirect Mission teams from JPL, LaRC, JSC, and KSC for their inputs.

\section{References}

Orion Multi-Purpose Crew Vehicle (MPCV) Program Operations Concept (OPSCON) Document, MPCV72093, Rev.A, 2013.

Constellation Program Level II Coordinate Systems, CxP-70138, Rev. B, 2009. 